\title{
TESSX: A Mission for Space Exploration with Tethers
}

\author{
Mario L. Cosmo and Enrico C. Lorenzini. ${ }^{\dagger}$ \\ Harvard-Smithsonian Center For Astrophysics, Cambridge, Massachusetts, 02138 \\ Daniel J. Gramer \\ Orbital Technologies Corporation, Madison, Wisconsin, 53717 \\ John H. Hoffman ${ }^{\S}$ \\ The University of Texas, Dallas, Texas, 75080 \\ and \\ Andre P. Mazzoleni" \\ North Carolina State University, Raleigh, North Carolina, 27695
}

\begin{abstract}
Tethers offer significant potential for substantially increasing payload mass fraction, increasing spacecraft lifetime, enhancing long-term space travel, and enabling the understanding and development of gravity-dependant technologies required for Moon and Mars exploration. The development of the Tether Electrodynamic Spin-up and Survivability Experiment (TESSX) will support applications relevant to NASA's new exploration initiative, including: artificial gravity generation, formation flying, electrodynamic propulsion, momentum exchange, and multi-amp current collection and emission. Under the broad term TESSX, we are currently evaluating several different tether system configurations and operational modes. The initial results of this work are presented, including hardware development, orbital dynamics simulations, and electrodynamics design and analysis.
\end{abstract}

\section{Introduction}

Tethers will play an important role in NASA's new space exploration initiative ${ }^{1}$. The Tether Electrodynamic Spin-up and Survivability Experiment (TESSX) is a spinning tethered space-borne system, consisting of two endmasses separated by an electrodynamic tether. TESSX will explore the applicability of spinning electrodynamic tethers to the following enabling technologies critical to NASA's Lunar and Planetary Exploration Initiative:

Artificial Gravity: The Tethered Artificial Gravity Spacecraft (TAGS) consists of two end-masses connected by a tether. The system is designed to be spun-up in space and generate centripetal forces, allowing a range of gravity levels to be realized. There are two major TAGS application areas. The first one is reducing astronaut bone and muscle loss during long-term space missions. The effects of weightlessness on humans have been extensively studied since the beginning of space exploration, and long duration space flights are one of the initiative's main goals. The second TAGS application area is the generation of a prescribed gravity level to simulate Lunar $(1 / 6 \mathrm{~g})$, Martian (3/8g), and other gravity environments for technology and hardware development (such as for in-situ utilization systems), and for studying the biological affects of reduced gravity levels.

\footnotetext{
*Astrophysicist, Radio And Geoastronomy Division, MS 80.

${ }^{\dagger}$ Space Scientist, Radio And Geoastronomy Division, MS 80, Senior AIAA Member.

‡Principal Mechanical Engineer, Senior AIAA Member.

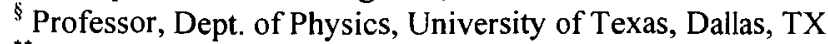

${ }^{* *}$ Associate Professor, Dept. of Mechanical and Aerospace Engineering, Box 7910, AIAA Associate Fellow.
} 
Formation Flying: Tethers can be the structural elements and control actuators at the same time. As structural components, tethers can save propellant mass by keeping the formation together in both open (i.e. pearls-on-astring) and closed (i.e. spinning loops) configurations. As control actuators, tethers will: (a) control overall formation, (b) increase formation stability and control redundancy (e.g. thruster failure), (c) minimize propellant use during on-station and re-targeting maneuvers, (d) control the attitude of each spacecraft in both pitch and roll, and (e) change the baseline formation by just reeling tether in or out ${ }^{3,4}$.

Momentum Transfer: Large spinning systems can transfer momentum to payloads and impart velocities of the order of $\mathrm{km} / \mathrm{s}$. Momentum transfer systems offer an incredible potential for substantially increasing payload mass fraction, increasing spacecraft lifetime, and enhancing operational flexibility for a variety of applications for planetary exploration ${ }^{5,6}$.

Electrodynamic Propulsion: By interacting with the surrounding plasma, conductive tethers can produce electricity and generate a force to either boost or deboost a spacecraft. Bare tethers offer the most promising solution to collect multi-amp currents even under low plasma conditions and at lower costs both in terms of mass and architecture, as compared to other configurations ${ }^{7}$.

TESSX will also test other tether technologies, such as tether survivability and safety; collection and emission; and system deployment, spin-up, and control.

Thanks to the versatility of tethers, the features mentioned above can be combined and matched depending on the objectives of the mission under consideration. For example, consider an artificial gravity facility testing Mars' ISRU technologies in LEO. At the end of the test the products can be returned to Earth by using the tip velocity provided by the spinning tether, thus saving significant propellant mass.

TESSX hardware, software, analysis and other technologies will have direct implications on

spaceborne applications requiring both momentum transfer and artificial gravity. High-performance electrodynamic tethers for exploration will require multi-amp currents, surviving several years of exposure to the harsh space environment, and withstanding many kilovolts of electrical potential difference with respect to the surrounding plasma. The development of TESSX will space-qualify this technology and foster the advancement of tethers for space exploration.

Tethers are a well-established technology. A number of flights have shown that tethers are viable options for space transportation offering quite a wide range of benefits:

- Tether hardware can be made small and reliable

- If properly designed, tethers can resist the harsh space environment

- The dynamics of spinning tethers is inherently stable

- With the right numerical tools, tether dynamics can be predicted and controlled

\section{TESSX}

TESSX is currently being developed under a Phase II Small Business Technology Transfer (STTR) contract with NASA/MSFC by Orbital Technologies Corporation $\left(\right.$ ORBITEC $^{\mathrm{TM}}$ ), the Harvard-Smithsonian Center for Astrophysics, The University of Texas at Dallas, and North Carolina State University ${ }^{8,9}$. The STTR program fosters collaboration between small businesses and nonprofit research institutions to "move ideas from the laboratory to the marketplace, to foster high-tech economic development and to address the technological needs of the Federal Government".

TESSX is a spinning tethered system, consisting of two end masses separated by an electrodynamic tether, developed to demonstrate key space tether system technologies, including:

- Survivable multi-strand electrodynamic tethers, insensitive to micrometeoroid impacts

- High accuracy tether system tracking

- Multi-amp current collection

- Multi-amp current emission 
- Deployment, spin up and operation of a spinning tethered system

- Generation of a prescribed gravity level

- Multi-strand tether deployer and management system

- Integrated electrodynamic tether system operations.

Launch vehicle options are being evaluated for different TESSX-related missions. Launch variables considered include: payload mass, packaged volume and geometry, mass distribution, inserted orbit capability, launch costs, and launch opportunities. Where applicable, these parameters are being evaluated against the TESSX architecture and systems requirements to establish the launch approach that best fits each mission.

One launch approach that shows promise for smaller TESSX-related missions is the use an Expendable Launch Vehicle Secondary Payload Adapter ring (or ESPA ring). The ESPA system is a mechanical extension, below the primary payload, designed to accommodate secondary payloads on the Atlas $V$ and Delta IV Evolved Expendable Launch Vehicles (EELV). The ESPA ring was developed to maximize the abilities of EELV and provide an affordable option for delivering small satellites into space. Figure 1 illustrates the standard ESPA ring configured for six secondary payloads situated around the ring. However, it is envisioned that TESSX payloads may partially or wholly wrapped around the entire ring, and may be integrated as the only ESPA secondary payload for the launch. Larger TESSX-related payloads may benefit from the increased volume offered by the Pegasus XL, as a single or dual payload. We are also evaluating other launch opportunities slated to be available in the near future.

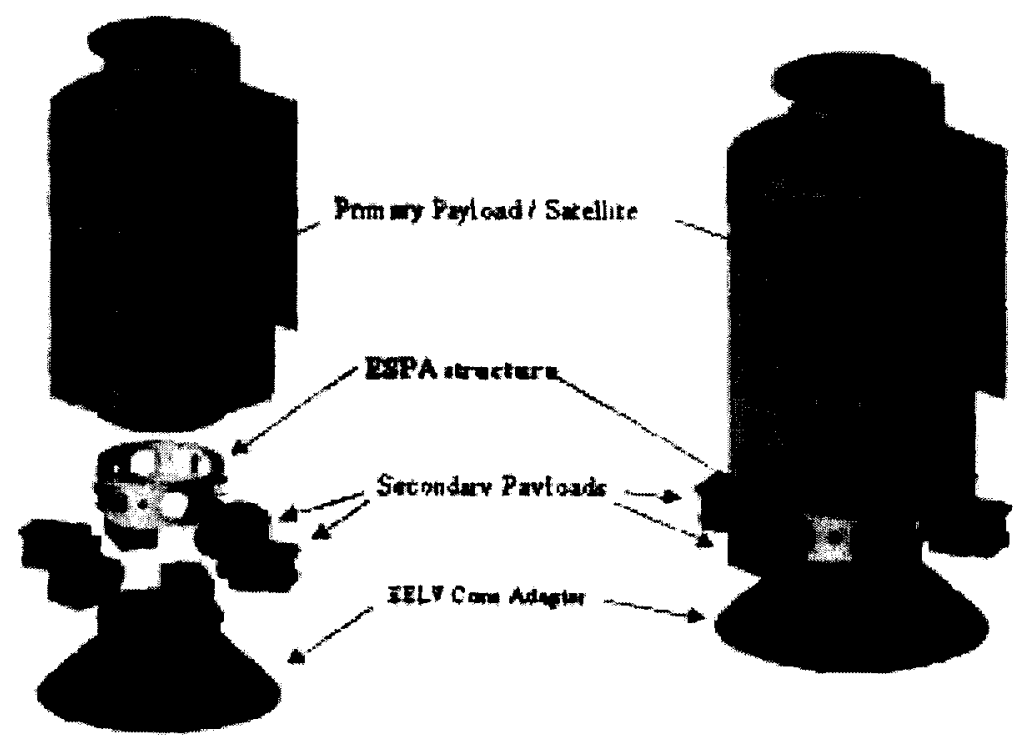

Figure 1. Fully Loaded ESPA Stack on EELV Illustration (Source: DoD Space Test Program, "Secondary Payload Planner's Guide For Use On The EELV Secondary Payload Adapter", November, 2004)

The deployer, currently being developed at University of Texas at Dallas, contains the tether and the mechanism to deploy it in a prescribed manner by either controlling the tension, the tether deployment rate, or the deployed length. A bail feeds the tethers evenly onto a spool during deployment and retrieval. Separate motors, controlled by microprocessors, drive the spool and the bail. Spools were originally sized to hold $2 \mathrm{~km}$ of Spectra tether. Other spool sizes are being considered to accommodate different tether lengths and designs of fail-safe multi-strand micrometeoroid survivable tethers ${ }^{10}$. Tether materials being considered are Kevlar and Spectra for the nonconductive section and fine stranded copper or aluminum for the conductive part.

The deployer system was originally developed to fiy in a Shuttle-based GAS-CAN to test deployment and retrieval of tethered payloads in space. Two sets of deployers and drive pulleys were built to be mounted at each end of the GAS-CAN frame with respect to the center deck. Ground based tests with this system have been successful.

3

American Institute of Aeronautics and Astronautics 


\section{TESSX Design and Analysis}

Our initial work presented here has focused on the following design features:

- Systems consisting of two platforms: a 100kg-endmass attached to a $1000-\mathrm{kg}$ Ballast mass, such as a spent upper stage

- Tether lengths ranging from a few kilometers for formation flying and generation of artificial gravity, to tens of kilometers for momentum transfer

- Both bare tethers ${ }^{9}$ and metallic spheres have been considered as anodes in electrodynamic propulsion applications

- Orbital conditions that have been analyzed include LEO, GTO and earth trailing orbits

- Both thrust and drag systems.

In both cases, the tether is comprised of the following subsections:

- Bare metallic

- Insulated conductive

- Non-conductive.

Either subsection, conductive or not, needs a core material able to bear the mechanical loads. Kevlar or Spectra are being considered for the tether's load-bearing subsection and Aluminum for the conductor.

Materials for the insulators and coatings to protect the tether from the environment will be considered later on in the course of the investigation.

The tether mass is a function of the tether material, the ratio between the end-platform masses and the tip velocity $^{12}$. Weight could be saved by tapering the tether, although the tether design becomes more complicated.

We have analyzed two basic designs:

A. A system able to generate $1 \mathrm{~g}$, consisting of a $100-\mathrm{kg}$ end-mass and $1000 \mathrm{~kg}$ ballast separated by a $4.4 \mathrm{~km}-\mathrm{long}$ tether; this will require $2 \mathrm{~kg}$ of Spectra tether.

B. A system able to deliver $1 \mathrm{~km} / \mathrm{s}$ at its tip, consisting of a $100-\mathrm{kg}$ end-mass and $1000 \mathrm{~kg}$ ballast separated by a $40 \mathrm{~km}$-long tether; this will require $60-\mathrm{kg}$ of tapered tether or $70 \mathrm{~kg}$ of cylindrical tether.

The tether diameter is chosen considering the mechanical loads and the thermal inputs. Ohmic heating generated by multi-amp currents needs to be radiated away from the tether to prevent damaging the insulator and the load bearing materials. Figure 2 shows that in order to keep a multi-amp tether in Earth orbit at reasonably low temperatures, the diameter should be $3.5 \mathrm{~mm}$ or larger. Large diameters also imply larger conductive surfaces, at the cost of increasing the mass.

The mass properties of the conductor as well as the electrodynamic characteristics have been computed considering a $1000 \mathrm{~kg}$ system, a vertically stabilized tether and average ionospheric conditions (e.g. $5 \times 10^{11} \mathrm{~m}^{-3}$ ). We will show, however, that the changing orientation of the tether as it spins affects the values of the currents and electromotive force (EMF).

For the $1 \mathrm{~g}$-system we have considered a $4 \mathrm{~km}$ tape, $2.5 \mathrm{~mm}$ wide and $0.5 \mathrm{~mm}$ thick, yielding about $20 \mathrm{~m}^{2}$ of bare area and generating a maximum drag of $0.3 \mathrm{~N}$. The conductor adds $11 \mathrm{~kg}$ to the mass budget and only the last $200 \mathrm{~m}$ of tether closest to the cathode is insulated.

The $1 \mathrm{~km} / \mathrm{s}$-system, on the other hand, has $20 \mathrm{~km}$ of bare aluminum tape, $5 \mathrm{~mm}$ wide and $0.5 \mathrm{~mm}$ thick, attached in series to a $20 \mathrm{~km}$ non-conductive Spectra tether. The bare area is $200 \mathrm{~m}^{2}$ and the conductor adds about $100 \mathrm{~kg}$ to the mass budget. A schematic of the system is shown in Figure 3. 


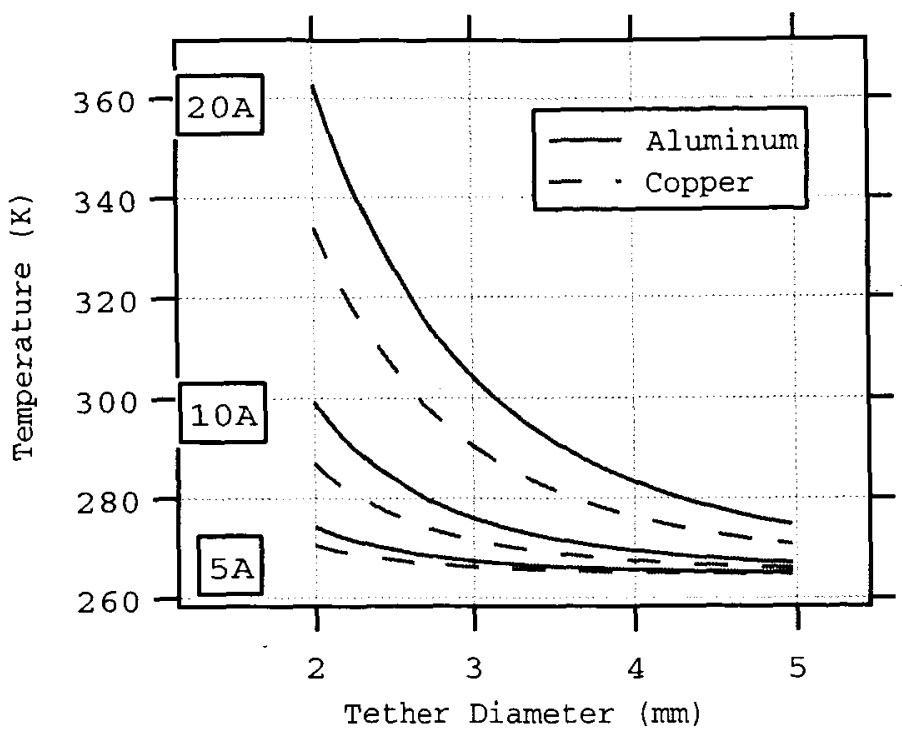

Figure 2. Tether Equilibrium Temperature Vs. Diameter

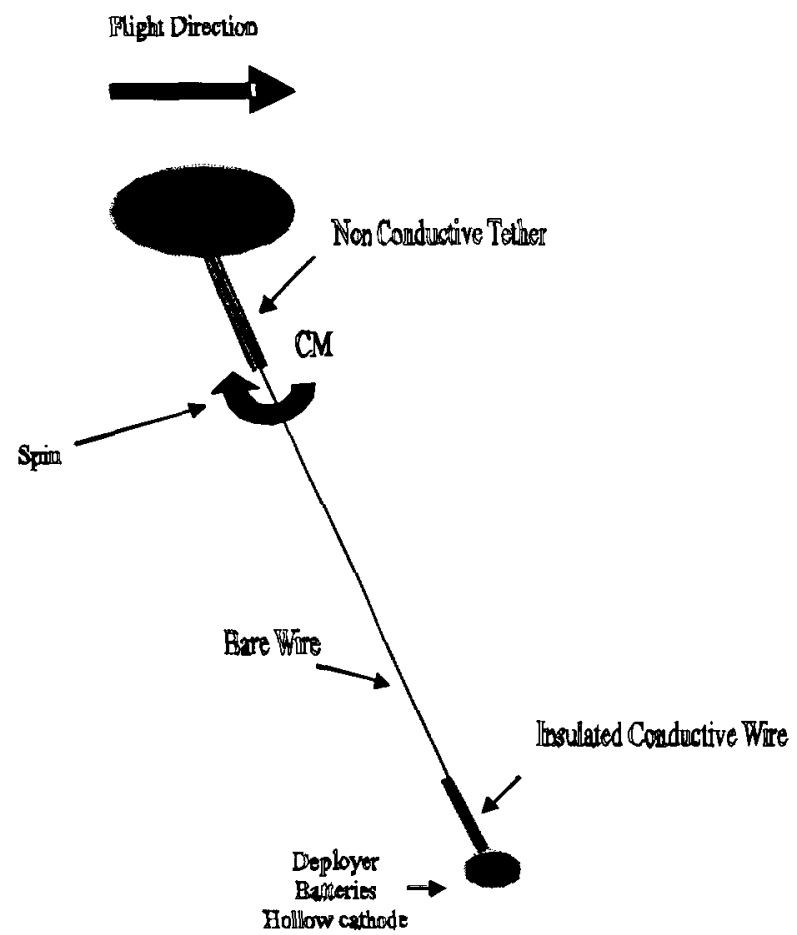

Figure 3. Schematic for a TESSX Demo Flight (dimensions not to scale)

In our simulations, we have considered bare tethers as anodic terminations. Our trade study has considered both drag and boost configurations; however, we have concentrated on a demo flight utilizing a drag system. The Drag system offers a simpler design in terms of mass and subsystems. This system is suitable for a demo flight, if longterm tether survivability beyond about 60 days is not a mission objective unless the system is placed in a GTO orbit. 
SAO high-fidelity numerical code has been used to analyze various TESSX architectures. The code has been extensively used over the years to study the design and the feasibility of tethered systems as well as being thoroughly validated with flight data from several missions.

Both platforms and tethers are discretized by mass elements, which are acted upon by the external forces and various thermal inputs.

The code has a suite of very sophisticated environmental models, namely: MSIS atmospheric model, IRI and IGRF for plasma density and geomagnetic field for the calculation of Lorentz's forces, central and higher harmonics of the earth's gravitational field, luni-solar attraction and solar pressure.

The thermal model utilizes the solar direct illumination, the Earth's IR radiation and albedo, ohmic heating and the tether's emitted and absorbed radiation. Temperature affects the lengths of the tether segments as well as their electrical resistances.

The artificial gravity system is in a slightly eccentric orbit $(300 \times 1000 \mathrm{~km})$ at $0^{\circ}$ inclination. Figure $4(\mathrm{a})$ shows that the ED excitation takes about four hours to start spinning the system and six days to reach the final rate that provides $1 \mathrm{~g}$ acceleration. At the same time, the system altitude drops by about $90 \mathrm{~km}$ at a constant rate of about 15 $\mathrm{km} /$ day as shown in Fig. 4(b). The correlation between the current collected by the bare tether and the electron density and EMF are shown in figure 4(c) and 4(d), respectively. The system is able to collect currents even when the density drops by one or two order of magnitude. In this case the current is about $50 \%$ off the peak. There is no current collection when the tether is horizontal and the EMF goes to zero.

Figure 4(e) shows the comparison between the current collected by the bare tether system and an equivalent $10 \mathrm{~m}$-diameter metallic sphere. In the sphere case we have applied the Parker-Murphy model as adapted for the TSS1 and TSS-1R flights ${ }^{13}$ with the assumption that the bias voltage is $90 \%$ of the produced EMF. In actuality, the losses due to plasma grounding and ohmic resistance are higher and the bias voltage would be significantly less than the value we have used.

The electrodynamic versatility of the bare-tether system is more apparent in the case of the GTO orbit where the system dips into a significant ionosphere for a very brief time as compared to the orbital period. The system is able to collect tens of amperes even when the density drops by 1-2 orders of magnitudes.

Figures $5(\mathrm{a}-\mathrm{c})$ show the performance of the $1 \mathrm{~km} / \mathrm{s}$ tether where there is still significant collection at low plasma densities. The comparison with a $25 \mathrm{~m}$-diameter sphere shows that the bare tether can collect $50 \%$ or more due to its electrodynamic characteristics.

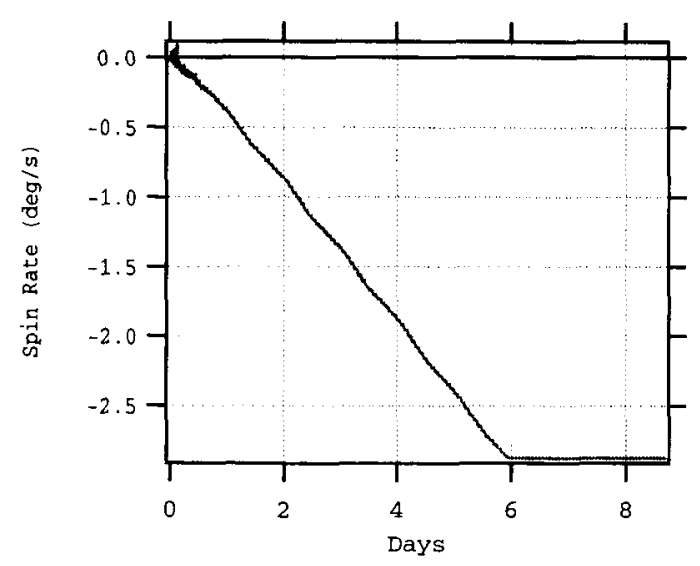

Figure 4(a). Spin Rate Vs. Time

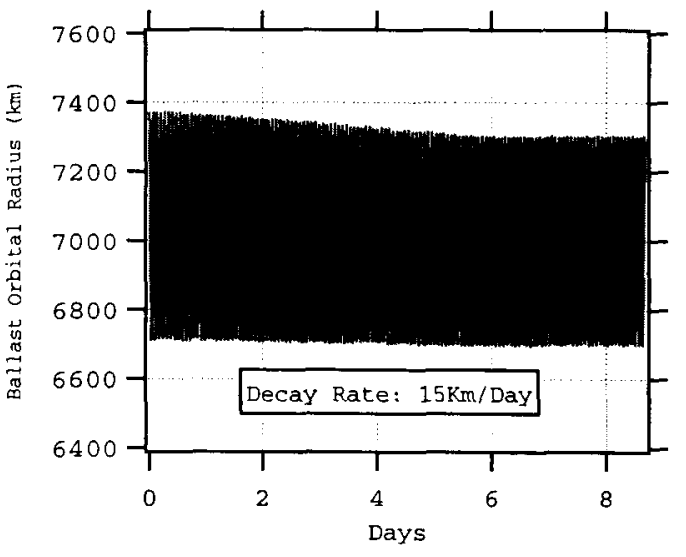

Figure 4(b). Ballast Orbital Radius Vs. Time 


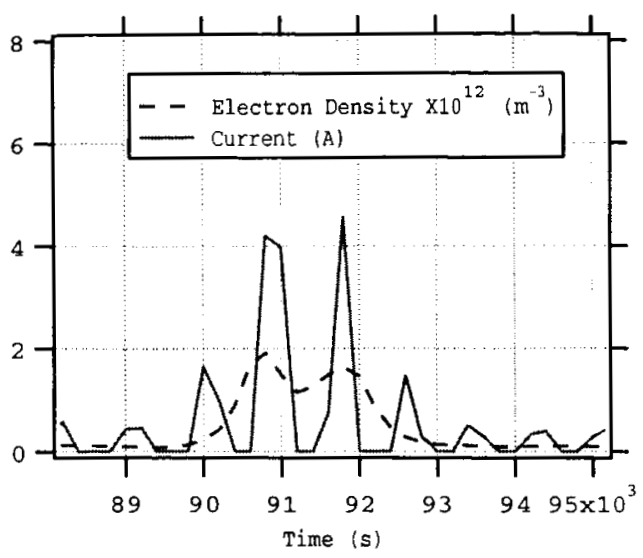

Figure 4(c). Electron Density and Current vs. Time

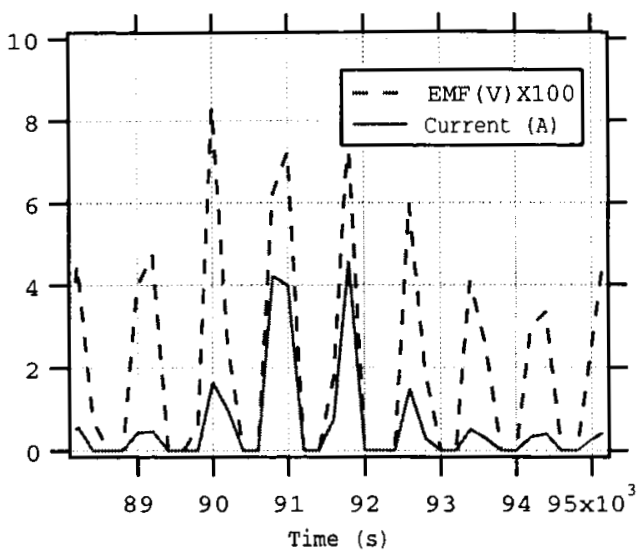

Figure 4(d). EMF and Current vs. Time

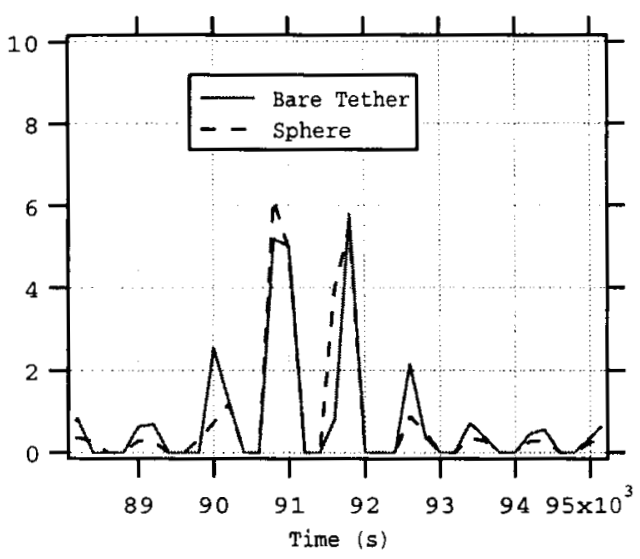

Figure 4(e). Currents collected by Bare Tether and 10m-Diameter Metallic Sphere vs. Time

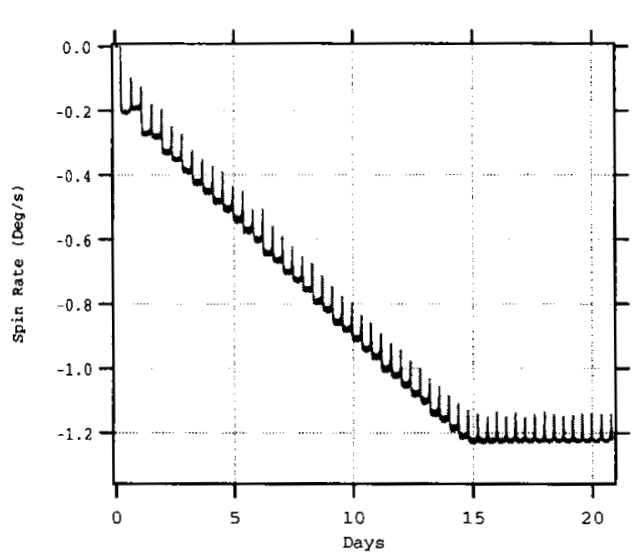

Figure 5(a). GTO Orbit - Spin rate vs. Time

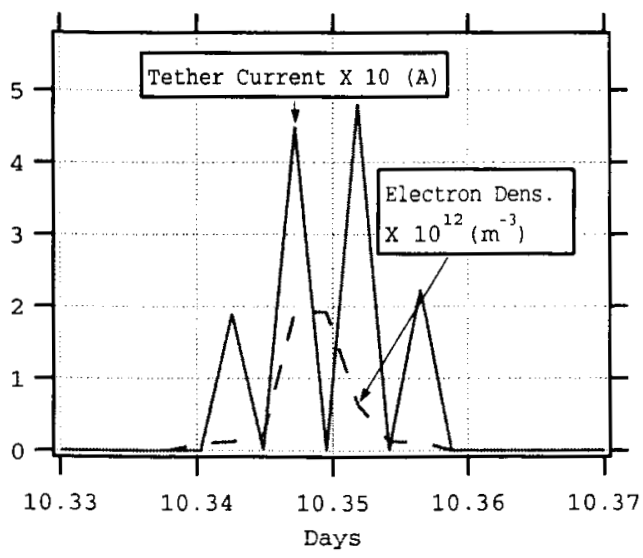

Figure 5(b). GTO Orbit - Electron Density and Bare Tether Current vs. Time

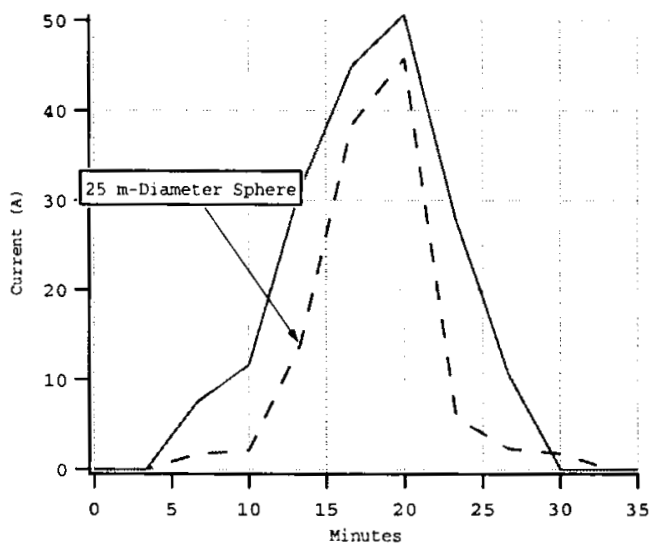

Figure 5(c). GTO Orbit - Currents Collected by a Bare Tether and $25 \mathrm{~m}$-Diameter Sphere 
The tremendous benefits of In-Situ Resource Utilization (ISRU) for planetary exploration and operations have been widely published, and are generally accepted. However, there are several critical technologies required to successfully implement ISRU in reduced gravity environments. Many of these technologies involve gravitydependant hardware and processes which need to be validated under realistic gravity conditions for relatively long periods of time. Reliable development of efficient ISRU hardware will remain extraordinarily difficulty until a better understanding of reduced gravity phenomena has been established. A system such as TAGS provides a reduced gravity platform for this purpose "close to Earth", rather than on the surface of the Moon or Mars, at greatly reduced expense. Figure 6 illustrates the spin rate required to simulate Lunar and Martian gravity levels as a function of the spinning tethered system length.

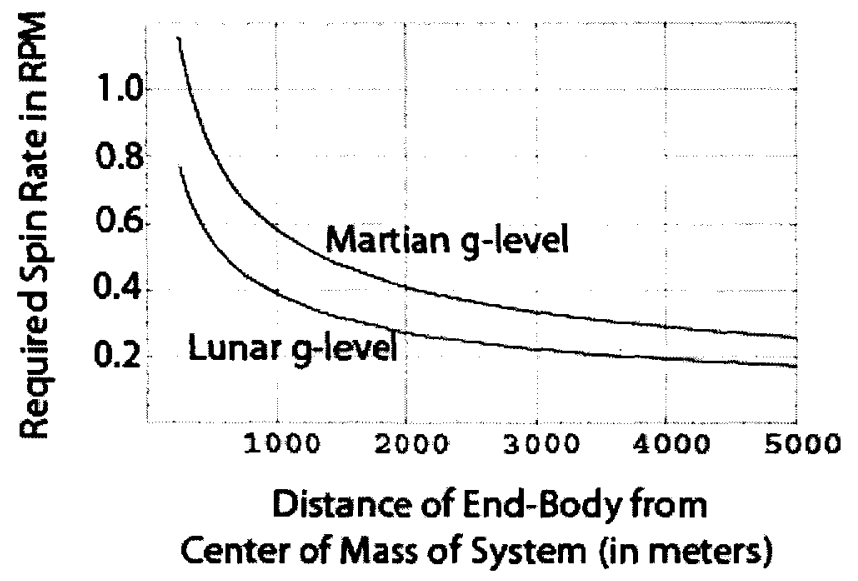

Figure 6. Spin Rate vs. Tether Length Required to Simulate Lunar and Martian Gravity Levels (tether length will be a function of mass distribution of the two end-bodies, e.g. if the end-bodies are of equal mass, then the tether length will be twice the distance of the each-body from the center of mass)

\section{Conclusion}

Orbital Technologies Corporation, the Harvard-Smithsonian Center for Astrophysics, The University of Texas at Dallas, and North Carolina State University are involved in a cooperative effort to develop tethered system technologies that support applications relevant to NASA's new exploration initiative, including: artificial gravity generation, formation flying, electrodynamics propulsion, momentum exchange, and multi-amp current collection and emission. The TESSX project will be a groundbreaker for several technologies critical to the successful implementation of NASA's new initiative. In this paper, we have presented two candidate designs that show great promise for the generation of artificial gravity and momentum transfer. The system feasibility has been positively assessed and we are currently proceeding with more detailed TESSX spacecraft design and analysis work. In the course of this work we will be looking more closely at the system stability during spin-up and long-term operations, as well as to the quality of the $g$ accelerations for the testing of In-Situ Resource Utilization (ISRU) and the biological effects of reduced g-levels. Multi-strand tether deployer development and testing, tether-dynamics ground experiments, and mission planning will also be completed.

\section{Acknowledgments}

This work is being conducted under a Phase II STTR with NASA Marshal Space Flight Center. The authors wish to thank the In-Space Propulsion Program, Kirk Sorensen, the NASA Technical Monitor, and Joe Bonometti (NASA/MSFC) for their support and technical guidance. 


\section{References}

'Steidle, C.E., "Office of Exploration Systems: Program Overview", March 2-3, 2004

${ }^{2}$ Gilruth, R.R."Manned Space Station-Gateway to Our Future in Space." Manned Laboratories in Space, S.F.Singer, Ed., D.Reidel Publishing Company, 1968, 1-10

${ }^{3}$ Cosmo, M.L., Lorenzini, E.C., and Bombardelli, C., "Space Tethers as Testbeds for Spacecraft Formation-Flying," $14^{\text {th }}$ AAS/AIAA Space Flight Mechanics Conference, Maui, HI, February 8-12, 2004

${ }^{4}$ Bombardelli, C., Lorenzini, E.C., and M.B.Quadrelli. "Re-Targeting Dynamics of a Linear Tethered Interferometer," Journal of Guidance, Control and Dynamics, Vol.27, No.6, 2004, 1061-1067

${ }^{5}$ Lorenzini, E.C., Cosmo, M.L., Kaiser, M., Bangham, M.E., Vonderwell, D.J., and Johnson, L., "Mission Analysis of Spinning Systems for Transfers from Low Orbits to Geostationary." Journal of Spacecraft and Rockets, 2002, Vol. 37, No. 2, 165-172.

${ }^{6}$ Hoyt, R.P., "Momentum Exchange Tether Principles, "Space Technology \& applications International Forum (STAIF2002), Albuquerque, New Mexico, 2002

7 Estes, R.D. , Lorenzini, E.C. , Sanmartin, J., Pelaez J., Martinez-Sanchez, M. and Johnson, L. "Bare Tethers for Electrodynamic Spacecraft Propulsion," Journal of Spacecraft and Rockets, Vol. 37, No. 2, 205-211, 2000.

${ }^{8}$ Rice, E.E., Gramer, D.J., Hoffman, J., Mazzoleni, A., Knuth W.H., Jordan, J., "Phase I Final Report on Tethered Artificial Gravity Spacecraft (TAGS)", OTC-GS-132-FR-04-1, NASA Contract NAS8-03041, Orbital Technologies Corporation (ORBITEC $^{\text {TM}}$ ), Madison, WI, 13 January 2004.

${ }^{9}$ Mazzoleni, A. P. , and Hoffman, J.H., "Overview of the Tethered Artificial Gravity Satellite Program", AAS Paper, 02-204, AAS/AIAA Space Flight Mechanics Meeting, San Antonio, TX, January 28-30 2002

${ }^{10}$ Forward, R.L., Hoyt, R.P., "Failsafe Multiline Hoytether Lifetimes," AIAA Paper 95-2890, 31 ${ }^{s t}$ AIAAA/SAE/ASME/ASEE Joint Propulsion Conference, San Diego, CA, July 1995

"SanMartin, J.R., M.Martinez-Sanchez, and E.Ahedo, "Bare Wire Anodes for Electrodynamic Tethers," Journal of Propulsion and Power, Vol.9, No.3, 1993, 353-360

${ }^{12}$ Lorenzini, E.C.,"'Error-Tolerant Technique For Catching a Spacecraft With a Spinning Tether," Journal of Vibration and Control, 10, 2004, 1473-1491

${ }^{13}$ Vannaroni, G, M. Dobrowolny, J.P. LeBreton, E. Melchioni, F. De Venuto, C.C. Harvey, L. Iess, U. Guidoni, C.Bonifazi, F. Mariani. "Current-Voltage Characteristics of the TSS-IR satellite: Comparison with Isotropic and Anisotropic Models." Geophysical Research Letters, 25, Issue 5, 1998, 749-752 